\title{
Autonomous Tuning Methods for Piezoelectric Energy Harvesting Generators
}

\author{
Loreto Mateu, Johannes Knauer, Peter Spies and Henrik Zessin ${ }^{1}$ \\ ${ }^{1}$ Fraunhofer Institute for Integrated Circuits IIS, Nordostpark 84, 90411 Nuremberg, Germany \\ loreto.mateu@iis.fraunhofer.de
}

\begin{abstract}
This paper explores two different methodologies that are able to change the resonance frequency of a piezoelectric cantilever. In real-life energy harvesting applications like logistics, condition or structural health monitoring, the ambient frequency is changing. A drawback of harvesting energy from ambient vibrations is the fact that the frequency of the ambient vibration and the resonance frequency of the piezoelectric generator should match. Therefore, in order to sufficiently and continuously harvest energy from ambient vibrations, it is necessary to be able to change the resonance frequency of the piezoelectric generator. To overcome this challenge, two frequency tuning methodologies that can be applied autonomously in operation are presented. A $10 \%$ frequency tuning is desired for example by applications in logistics.
\end{abstract}

Key words: energy harvesting, piezoelectric generator, frequency tuning, resonance frequency.

\section{Introduction}

A summary of tuning methodologies is given in [1] where either manual or autonomous methods are described. The ambient frequency with higher acceleration amplitude does not remain constant with time [2]. Therefore, for a real application, it is necessary to be able to tune the resonance frequency autonomously during operation.

The tuning concepts presented in the paper are suitable for this variant. Both methods use a piezoelectric bimorph cantilever as energy harvesting generator.

Following the generic model based on inertial kinetic energy harvesting developed by Williams and Yates [1], the natural resonance frequency of a piezoelectric energy harvester is:

$\omega_{n}=\sqrt{\frac{k}{m}}$

where $k$ is the stiffness and $m$ the weight of the harvester.

Therefore, the natural resonance frequency can be changed by modifying the stiffness $k$ of the harvester. The first method presented in this paper modifies the stiffness of the harvester by connecting a parallel capacitor to one piezoelectric layer of a bimorph cantilever. This methodology has been presented previously in [3]. Nevertheless, the fact that the frequency tuning works in the tuning range between the resonance and anti-resonance frequency has not yet been pointed out.
The second method for tuning the resonance frequency of a piezoelectric presented in the paper employs a magnetic force. The magnetic force applied changes by varying the rotation angle of the magnet [4], see Fig. 1, and not the distance between magnets [1], to influence the stiffness of the cantilever and therefore its resonance frequency. This method reduces the required volume for the generator compared to [1].

The tuning ratio of a mechanical harvester is given by eq. (2) [3].

Tuning ratio $=\frac{f_{r, \max }-f_{r, \min }}{f_{r, \min }} 100$

where $f_{r, \max }$ is the maximum resonance frequency and $f_{r, \min }$ is the minimum resonance frequency. The tuning ratio is a figure of merit to characterize the tuning capabilities as a ratio and not as an absolute number.

The paper concludes with a summary of the results and highlights the work still pending.

\section{Capacitive Tuning Methodology}

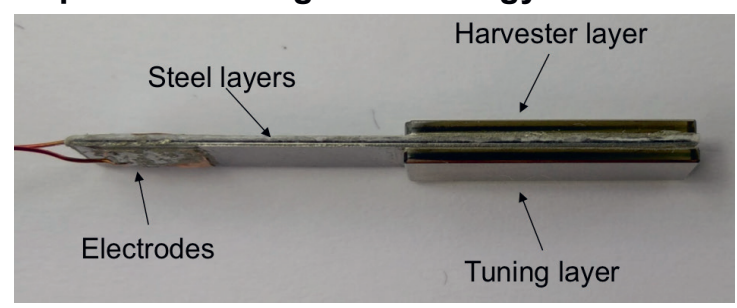

Fig. 1 Piezoelectric bimorph with PZN-5.5\%PT 
Fig. 1 shows the bimorph cantilever structure, employed for the capacitive frequency tuning. One piezoelectric layer is used for harvesting and the other one is used for frequency tuning by connecting it to an external capacitor $C_{s}$. By connecting $C_{s}$ to the piezoelectric cantilever, the parallel resonance frequency [5] is modified as described by eq. (3).

$$
f_{p}=\frac{1}{2 \pi \sqrt{L_{1} C_{1}\left(C_{p}+C_{s}\right) /\left(C_{1}+C_{p}+C_{s}\right)}}
$$

where $L_{1}$ is the motional inductance in the equivalent electric circuit, $C_{1}$ is the motional capacitance in the equivalent electric circuit, $C_{p}$ is the parallel capacitance in the equivalent electric circuit and $C_{s}$ is the shunt electrical capacitance added to the piezoelectric harvester for tuning purposes.

The piezoelectric material employed is $\mathrm{PZN}-5.5 \% \mathrm{PT}$ and free samples were provided by [6]. The dimensions of the cantilever beam are summarized in Tab. 1.

Tab. 1: Materials and dimensions employed for the bimorph piezoelectric cantilever with capacitive tuning

\begin{tabular}{|c|c|c|}
\hline & Material & $\begin{array}{c}\mathrm{L} \times \mathrm{W} \times \mathrm{T} \\
(\mathrm{mm})\end{array}$ \\
\hline $\begin{array}{c}\text { Piezoelectric } \\
\text { element }\end{array}$ & $\begin{array}{c}\mathrm{PZN}- \\
5.5 \% \mathrm{PT}\end{array}$ & $15 \times 5 \times 0.8$ \\
\hline Shim layer & Steel & $30 \times 5 \times 0.2$ \\
\hline Electrode & Copper & $5 \times 5 \times 0.07$ \\
\hline Conductive layer & CW2400 & $25 \times 5 \times 0.04$ \\
\hline $\begin{array}{c}\text { Non-conductive } \\
\text { layer }\end{array}$ & $\begin{array}{c}\text { Super } \\
\text { glue }\end{array}$ & $40 \times 5 \times 0.08$ \\
\hline
\end{tabular}

Fig. 2 shows the admittance of the tuning layer of the bimorph piezoelectric cantilever. $f_{m}$ is the frequency at maximum admittance whereas $f_{n}$ is the frequency at maximum impedance [6]. The value of $f_{n}$ is close to $f_{p}$ and frequency $f_{m}$ is close to $f_{s}$, motional series resonance frequency, eq. (4).

$$
f_{s}=\frac{1}{2 \pi \sqrt{L_{1} C_{1}}}
$$

Tab. 2 shows the values for $f_{m}, f_{s}, f_{n}$ and $f_{p}$ obtained without $C_{s}$, open circuit, and with different values of $C_{s}$ measured at the tuning layer of the bimorph cantilever. The tuning ratio of this tuning method depends on the distance between $f_{m}$ and $f_{n}$ since adding $C_{s}$ moves $f_{n}$ towards $f_{m}$. Capacitive tuning can only be performed with piezoelectric materials that provide values of $f_{m}$ and $f_{n}$. The frequency at which the maximum power is achieved at the piezoelectric harvesting layer is somewhere between $f_{m}$ and $f_{n}$.

Fig. 3 shows the measured resonance frequency as a function of $C_{s}$ normalized to the piezoelectric capacitance $C_{p}$ with $10.5 \%$ frequency tuning achieved. $C_{p}$ is $3.4 \mathrm{nF}$, for the piezoelectric element under test of Tab. 1. As expected, a change in $C_{s}$ brings a change to the resonance frequency in a defined region. For the cantilever bimorph of Fig. 1, the resonance frequency can vary from $441.5 \mathrm{~Hz}$, if the tuning layer is in open circuit, to $399.5 \mathrm{~Hz}$, if $C_{s}=$ $200 \mathrm{nF}$ is connected to the tuning layer.

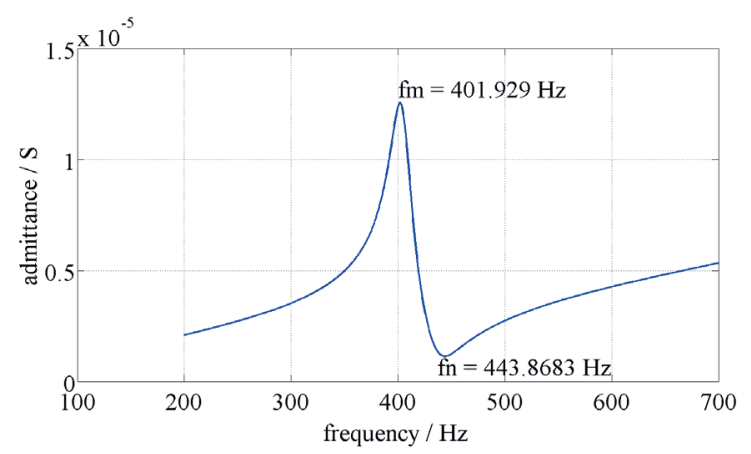

Fig. 2. Admittance as function of frequency for the piezoelectric harvesting layer of the piezoelectric bimorph cantilever

Tab. 2: Maximum admittance $f_{m}$ and maximum impedance $f_{n}$ values with different $C_{s}$

\begin{tabular}{|c|c|c|c|c|}
\hline $\mathrm{C}_{\mathrm{s}}(\mathrm{nF})$ & $\mathrm{f}_{\mathrm{m}}(\mathrm{Hz})$ & $\mathrm{f}_{\mathrm{s}}(\mathrm{Hz})$ & $\mathrm{f}_{\mathrm{n}}(\mathrm{Hz})$ & $\mathrm{f}_{\mathrm{p}}(\mathrm{Hz})$ \\
\hline without $\mathrm{C}_{\mathrm{s}}$ & 401.93 & 404.88 & 443.87 & 441.10 \\
\hline 0.535 & 403.78 & 407.13 & 436.82 & 433.15 \\
\hline 0.954 & 403.10 & 406.96 & 432.88 & 428.68 \\
\hline 2.2 & 401.82 & 406.93 & 426.45 & 421.05 \\
\hline 8.25 & 399.95 & 407.01 & 419.52 & 412.04 \\
\hline 142 & 402.90 & 406.95 & 409.3 & 406.95 \\
\hline 174 & 405.10 & 406.88 & 407.70 & 406.88 \\
\hline
\end{tabular}

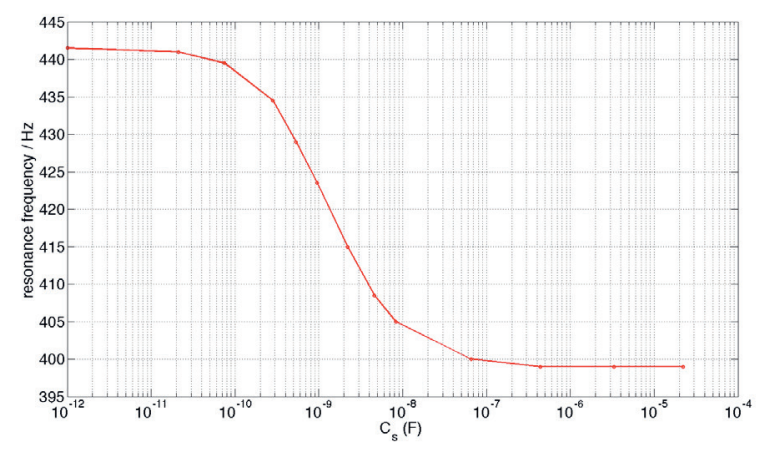

Fig. 3. Resonance frequency as a function of the ratio between capacitance $C_{s}$ and piezoelectric electrical capacitance $C_{p}$.

\section{Capacitive Self-tunable piezoelectric harvester}

A frequency tuning algorithm must be able to tune the resonance frequency of the piezoelectric harvester to the ambient frequency. Some tuning algorithms $[7,8]$ employ the piezoelectric voltage as input parameter. When either the voltage or the power reach their maximum value, it is assumed that the piezoelectric harvester is tuned. This methodology has been used for the algorithm of the capacitive self-tunable piezoelectric harvester.

Values of $\mathrm{C}_{\mathrm{s}}$ between $75 \mathrm{pF}$ and $65.6 \mathrm{nF}$ are inside the linear region of the curve shown in Fig. 3. 


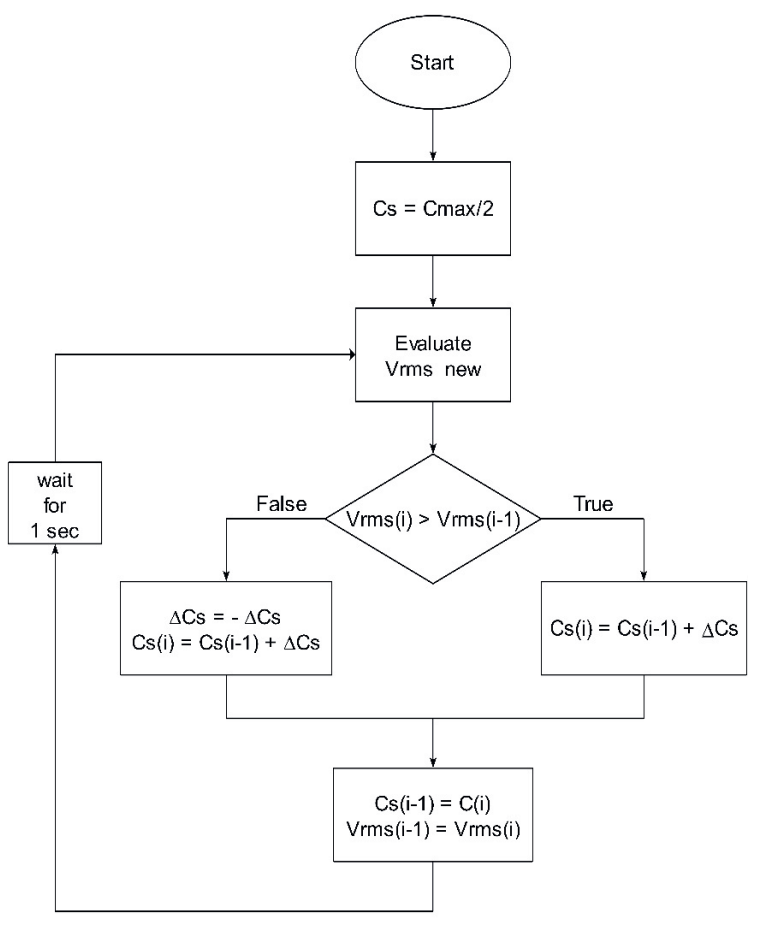

Fig. 4. Flowchart of the perturb and observe algorithm for frequency tuning

A perturb and observe algorithm [9], see Fig. 4, has been implemented with a DS1104 dSPACE R\&D controller board in Simulink. The algorithm starts at half the value of the maximum value of $C$ s inside the linear region, $\mathrm{C}_{\max }$. An array of capacitors and switches were connected in parallel [1] and driven by the DS1104 board in order to connect the appropriate value of $\mathrm{C}_{\mathrm{s}}$ in parallel to the harvesting layer of the bimorph cantilever. The value of $\mathrm{Cs}$ is given by eq. (5).

$$
C_{s}=\sum_{i=0}^{N-1} 2^{i} C_{i}
$$

where $N=8$ bit, $C_{i}$ is the value of the capacitor connected in series with switch $i, \Delta C_{s}=2^{i} C_{i}$. For example, $\mathrm{C}_{0}=100 \mathrm{pF}$ and $\mathrm{C}_{7}=12.8 \mathrm{nF}$. Thus, the minimum value of $\mathrm{C}_{\mathrm{s}}$ is $100 \mathrm{pF}$ and its maximum value is $24.78 \mathrm{nF}$.

However, a method that maximizes the piezoelectric voltage is not suitable for frequency tuning when the acceleration amplitude of the ambient vibration changes with the time, which happens often in real applications, since the piezoelectric voltage will change too.

\section{Experimental Results for the Capacitive Self-tunable piezoelectric harvester}

Fig. 5. shows an experimental measurement of the capacitive tuning. Fig. 5(a) displays when the perturb and observe algorithm is started. Fig. 5(b) shows the ambient frequency applied to the harvester as a function of time. Fig. 5(c) shows the value of Cs selected by the tuning algorithm and connected to the tuning layer. The output power of the harvesting layer is represented both employing the perturb and observe algorithm for maximizing the rms voltage of the harvesting layer and without using it, see Fig. 5(d). The resistive load at which the harvested power is maximum remains constant for all the values of Cs. The ambient frequency decreases with time and the output power remains constant between 441.5 $\mathrm{Hz}$ and $399.5 \mathrm{~Hz}$ while the MPPT algorithm runs.
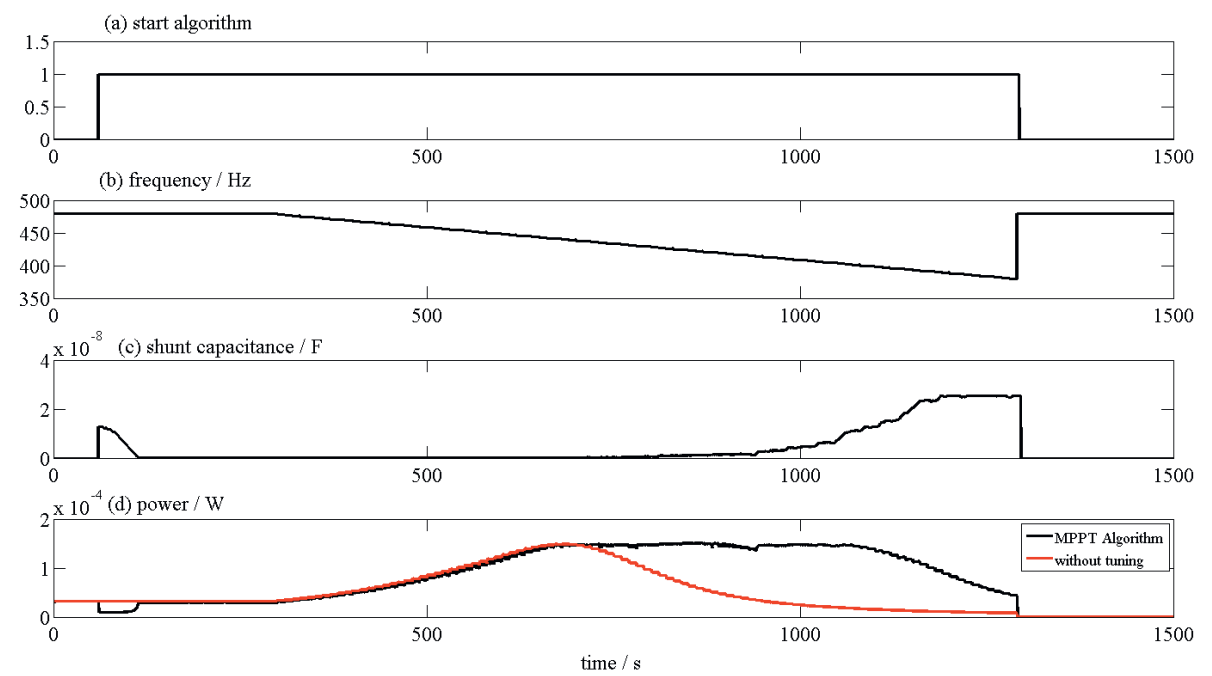

Fig. 5. Experimental measurement of the capacitive tuning, (a) indicates when the algorithm starts, (b) frequency of the ambient vibration, (c) shunt capacitance $C_{s}$, (d) output power of the harvesting layer with and without MPPT algorithm

\section{Magnetic Force Tuning Methodology}

The stiffness of the harvester can be changed by using a magnetic force, either attractive or repulsive and therefore a frequency tuning can be made.
Fig. 6 shows the structure employed for the bimorph tunable cantilever. Fixed magnets are attached to the upper and bottom end of the cantilever structure. The rotating magnets are located at a certain distance of 
the fixed magnets. The upper and bottom rotating magnets are moved at once by the same gear.

Fig. 7 shows that the frequency tunability has a linear region for an $\alpha$ angle between $30^{\circ}$ and $150^{\circ}$. The tuning ratio achieved with a magnet height of $3 \mathrm{~mm}$ and a distance between the fixed magnet at the cantilever and the rotating magnet of $8.5 \mathrm{~mm}$ is $9.8 \%$ for $0.1 \mathrm{~g}$ acceleration, see Fig. 8. This methodology can be implemented with any piezoelectric material. The output power is reduced by using a magnetic force since it adds a damping to the piezoelectric cantilever

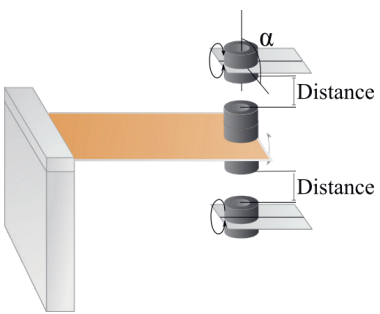

Fig. 6. Bimorph cantilever with magnetic tuning by rotating upper and bottom magnets

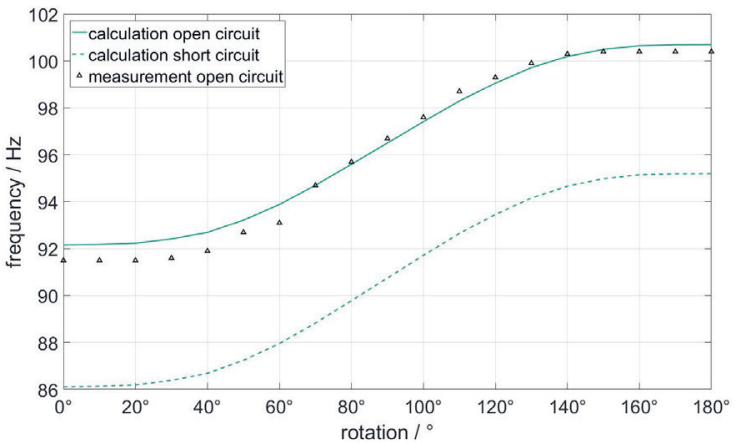

Fig. 7. Harvester frequency at different rotation angles of the magnets in open and short circuit with $0.1 \mathrm{~g}$ acceleration amplitude

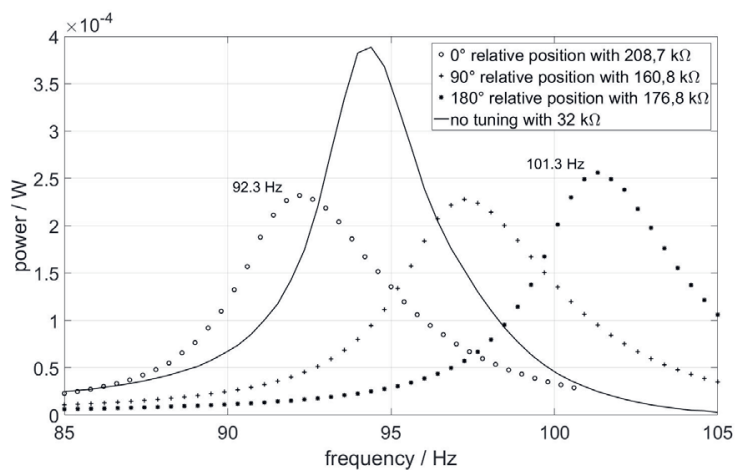

Fig. 8. Output power as a function of frequency without tuning and with an $\alpha$ rotation of the magnet at the optimum load

\section{Magnetic Force Self-tunable piezoelectric harvester}

Fig. 9 shows the set-up of the piezoelectric cantilever with the rotating magnets and a stepper motor. $64 \mathrm{~mJ} / \mathrm{Hz}$ are required for rotating $120^{\circ}$, between $30^{\circ}$ and $150^{\circ}$, the magnets and achieve $9.8 \%$ tuning ratio with the stepper motor selected. Thus, it is necessary to harvest energy for 42 minutes at the resonance frequency for rotating the magnets $120^{\circ}$.

The fact that the phase shift between the displacement of the harvester and the excitation displacement is $90^{\circ}$ at resonance has been used by $[10,11]$ in self-tuning algorithms. A PLL is used in [10] for determining the phase shift between both displacements and the algorithm compares the result with a reference voltage. An accelerometer and the piezoelectric harvester are employed in [11] for calculating the phase shift. A look-up table is employed by the tuning algorithm for achieving the resonance frequency of the harvester at the ambient vibration in [11]. The look-up table can be updated with new values. However, there is no information whether the tuning methods are robust against vibrations with more than one frequency component and with different vibration amplitudes.

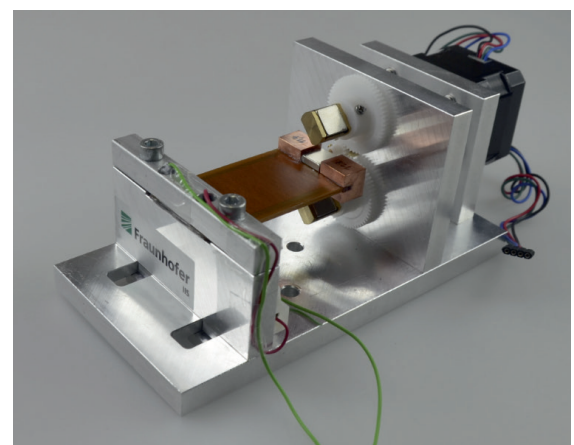

Fig. 9. Set-up of the piezoelectric harvester with stepper motor

The first issue to solve for the self-tuning of the piezoelectric harvester is how to detect that it is in resonance or how far away it is from resonance. The piezoelectric harvester is at resonance if its open circuit voltage and the acceleration of the ambient vibration have a phase shift of $90^{\circ}$ [12]. Thus, the open circuit voltage and the acceleration of the ambient vibration are the measured signals for calculating the ambient frequency and phase shift parameters needed for implementing the algorithm shown in Fig. 11. The algorithm drives the stepper motor that sets $\alpha$.

The frequency tuning algorithm has been implemented with the DS1104 dSPACE R\&D controller board in Simulink and drives a dual fullbridge LN298 board.
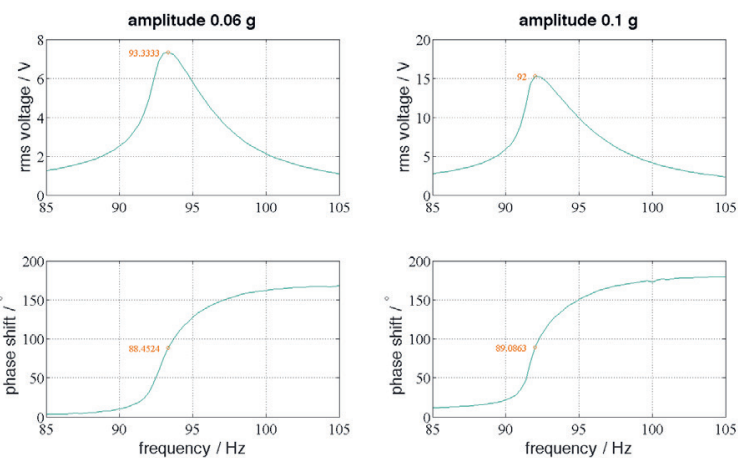

Fig. 10 Open circuit voltage of the piezoelectric harvester and phase shift between the open circuit 
voltage and the accelerometer response to the ambient vibration for a frequency range $85 \mathrm{~Hz}-105$ $\mathrm{Hz}$ for $\alpha=0^{\circ}$

Fig.10 shows the calculated phase shift between the open circuit voltage of the piezoelectric harvester and the ambient vibration frequency for a frequency sweep of the ambient frequency between $85 \mathrm{~Hz}$ and $105 \mathrm{~Hz}$ with $\alpha$ equal to $0^{\circ}$. The validity of this method is proven by plotting the effective voltage of the piezoelectric generator and corroborating that its maximum takes place at $90^{\circ}$ phase shift. Similar results have been obtained with $\alpha$ equal to $90^{\circ}$ and $180^{\circ}$.

In the range between $20^{\circ}$ and $155^{\circ}$ phase shift, there is a linear behaviour between the phase shift and the signal frequency, see Fig. 10 Thus, only in this phase shift range it is possible to determine if the resonance frequency of the piezoelectric harvester should be increased or decreased to match the ambient frequency and proceed to tune the resonance frequency.

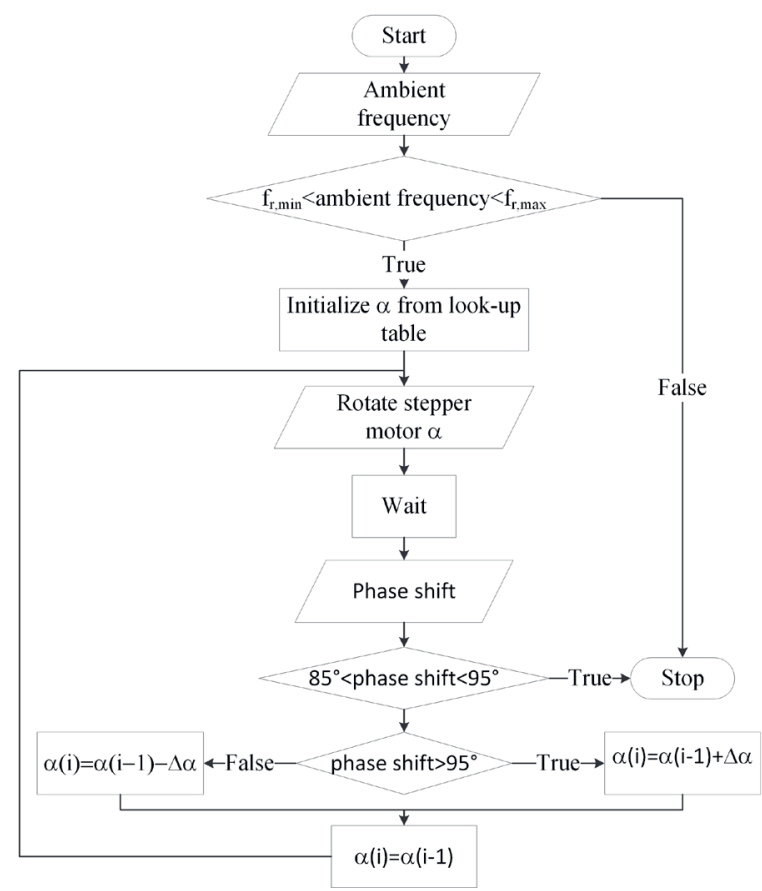

Fig. 11 Algorithm for the frequency tuning

The algorithm for the frequency tuning is represented in Fig. 11. If the ambient frequency is in the tuning range, the algorithm runs. A look-up table has been created and is used for setting the position of the magnet depending on the frequency. Therefore, the magnets are rotated and afterwards a perturb and observe algorithm is implemented until the resonance frequency matches the ambient frequency by detecting a $90^{\circ}$ phase shift.

\section{Experimental Results for the Magnetic Force Self-tunable piezoelectric harvester}

Fig. 12 shows the FFT of the acceleration signal employed for testing the self-tuning capability of the piezoelectric harvester. The acceleration signal is a combination of 6 different frequencies with a main signal of amplitude of $0.1 \mathrm{~g}$ and other 5 components with amplitudes up to $0.03 \mathrm{~g}$ and frequencies between $50 \mathrm{~Hz}$ and $150 \mathrm{~Hz}$. This experiment pursues to determine the robustness of the tuning algorithm

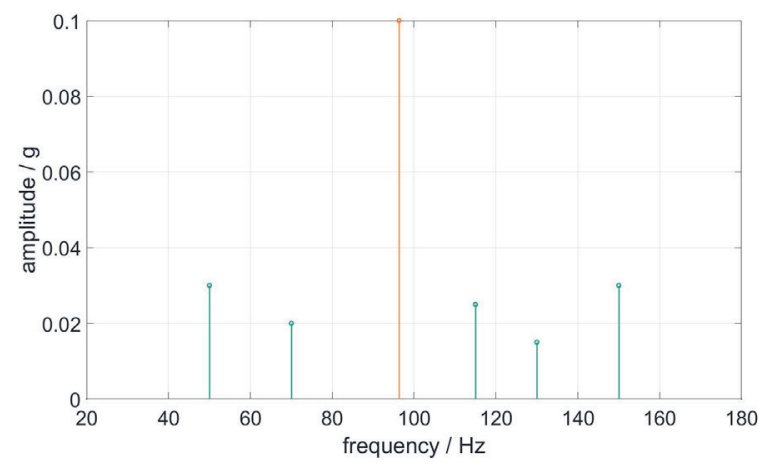

Fig. 12. FFT of the ambient acceleration employed for the tests summarized in Fig. 15

Fig.13 plots a summary of the experimental results with six different subplots. Fig.13-1) displays the ambient frequency of the main acceleration signal with amplitude $0.1 \mathrm{~g}$ fixed for the experiment (green) and the calculated frequency (orange). The tuning index indicates when the algorithm for the frequency tuning is running. If the tuning index is 0 , the tuning process is running while if the tuning index is set to 1 , the tuning process is finished.

First test is made with an ambient frequency of 95 $\mathrm{Hz}$. The magnets are rotated first with the data stored at the look-up table and afterwards $\Delta \alpha$ until the resonance is achieved with $\alpha=75^{\circ}$. Then, the tuning index is set to 1 . After $17 \mathrm{~s}$, the frequency of the main acceleration is fixed to $100 \mathrm{~Hz}$. Once the frequency tuning is activated, tuning index is 0 , the magnets are rotated to $\alpha=110^{\circ}$ according to the look-up table. Then, the magnets are rotated in $10^{\circ}$ steps until ca. $90^{\circ}$ phase shift is achieved. The ambient frequency is again changed from $100 \mathrm{~Hz}$ to $93 \mathrm{~Hz}$. The point in the tuning index indicates that the tuning algorithm is started. However, no tuning is possible since the error in the frequency calculation leads to a frequency value outside the frequency tuning range. At the last test, the main frequency increases to 96 $\mathrm{Hz}$. The algorithm starts and the magnets are rotated first to $\alpha=75^{\circ}$ and later to $\alpha=85$.

\section{Conclusions and Future Work}

The capacitive tuning moves $f_{n}$ towards $f_{m}$. The tuning ratio available for this methodology is limited to $\left(f_{n}-f_{m}\right) / f_{m}$ with the harvesting layer in open circuit. PZN-PT piezoelectric crystals have this property but in PZT crystals $f_{m}$ and $f_{n}$ are very close. Nevertheless, the magnetic tuning is not restricted to a certain kind of piezoelectric crystal.

Comparing both methods from the hardware side, the capacitive tuning requires just the control of several MOSFETs for the connection of the appropriate $C_{s}$ whereas in the magnetic tuning driving a stepper motor is needed.

The tuning algorithms should be programmed in a low-power microcontroller and tested. Further tests with real acceleration data should be done to get to know the limitations of the tuning algorithm. 
The capacitive tuning should be tested employing the algorithm based on phase shift between the acceleration of the ambient vibration and the piezoelectric harvester.

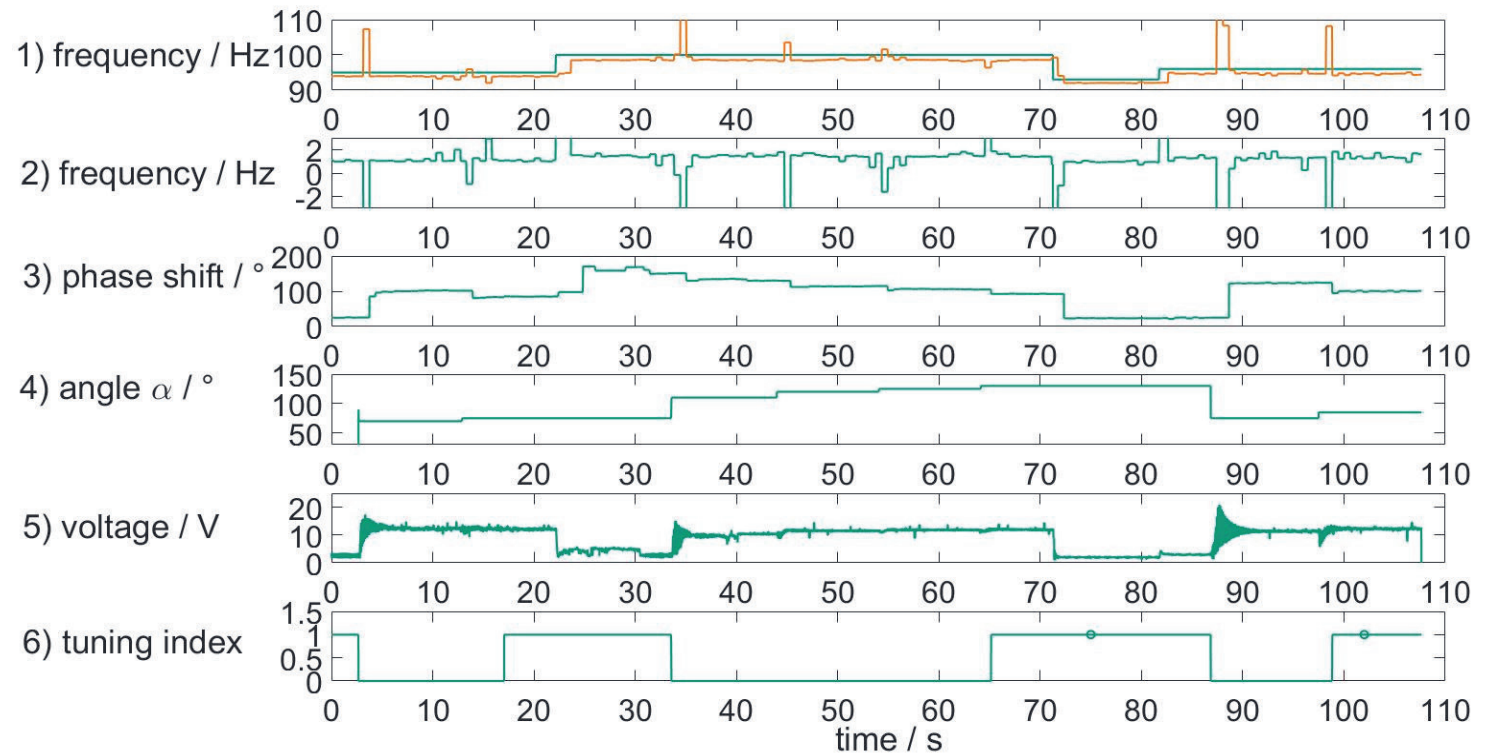

Fig. 13. 1) Frequency of the main ambient vibration signal (green) and measured frequency (orange), 2) frequency difference between the main signal and the measurement 1), 3) phase shift in ${ }^{\circ}$, 4) angle $\alpha$ of the magnets, in ${ }^{\circ}$, 5) open circuit voltage in $V$ and 6) tuning index

\section{Acknowledgements}

The authors gratefully acknowledge the financial support of the German Federal Ministry of Education and Research BMBF in the frame of CairGoLution project, Grant Number 13N12654 and the German Federal Ministry for Economic Affairs and Energy in the frame of the Daedalus project, Grant Number 50NA1311.

The authors would like to thank Davide Fabbri and Luca Martini for their great work at Fraunhofer IIS, during the realization of their Master Thesis, that has been partially used for this paper.

\section{References}

[1] S.W. Ibrahim, W.G. Ali, A review on frequency tuning methods for piezoelectric energy harvesting systems, Journal of Renewable and Sustainable Energy 4, 062703 1-29 (2012); doi $10.1063 / 1.4766892$

[2] X. Eguiluz, J. Legarda, L. Mateu, H. Zessin, P. Spies, Maximizing harvested energy for linear vibration-based generators, Procedia Engineering 120, 641-644(2015); doi: 10.1016/j.proeng.2015.08.693

[3] B.A. Seddik, G. Despesse, S. Boisseau, E. Defay, Strategies for Wideband Mechanical Energy Harvester, INTECH Open Access Publisher, 235-264 (2012); doi: 10.5772/51898

[4] L. Mateu, H. Zessin, P. Spies, Vorrichtung zur Umwandlung von mechanischer in elektrische Energie und entsprechendes Verfahren, International Patent Application PCT/EP2017/052151 (2017)
[5] Definitions, Standard, Methods of Measurement for Piezoelectric Vibrators“, IEEEStd 177, (1966)

[6] Microfine Materials Technologies PTE LTD, http://www.microfine-piezo.com

[7] D. Zhu, S. Roberts, M. Tudor, S. Beeby, Closed Loop Frequency Tuning of a Vibration-base Micro-generator, Proceddings of PowerMEMS 2008, 229-232(2008)

[8] V. Challa, M. Prasad, F. Fisher, Towards an autonomous self-tuning vibration energy harvesting device for wireless sensor network applications, Smart Mater. Struct. 20 (2011), doi: 10.1088/0964-1726/20/2/025004

[9] N. Femia, G. Petrone, G. Spagnuolo, M. Vitelli, Poer Electronics and Control Techniques for Maximum Energy Harvesting in Photovoltaic Systems, CRC Press, (2012), doi: 10.1109/MIE.2013.2272239

[10] C. Peters, D. Maurath, W. Schock, F. Mezger, Y. Manolis, A closed-loop wide-range tunable mechanical resonator for energy harvesting systems, Journal of Micromechanics and Microengineering 19, (2009), doi: 10.1088/09601317/19/9/094004

[11] C. Eichhorn, R. Tchagsim, N. Wilhelm, P. Woias, A smart and self-sufficient frequency tunable vibration energy harvester, Journal of Micromechanics and Microengineering, (2011), doi: 10.1088/0960-1317/21/10/104003

[12] S. Priya, D. Inman, eds., Energy harvesting technologies (Vol. 21), Springer, '(2009), doi: 10.1007/978-0-387-76464-1 